\title{
Emissionsoptimierung am hybriden Sattelzug mittels Most Relevant Testing
}

Hummel, Nicolas; Thiem, Mikula; Steinhaus, Tim et al.

(2020)

DOI (TUprints): $\quad$ https://doi.org/10.25534/tuprints-00014570

License:

only the rights of use according to UrhG

Publication type: Conference or Workshop Item

Division:

16 Department of Mechanical Engineering

Original source:

https://tuprints.ulb.tu-darmstadt.de/14570 


\section{Emissions-optimierung am hybriden Sattelzug mittels Most-Relevant-Testing}

Nicolas Hummel, M. Sc.; Mikula Thiem, M. Sc.; Tim Steinhaus, M. Sc.; Prof. Dr. techn. Christian Beidl

In Vergangenheit wurde am VKM eine Methode zur Identifikation emissionsrelevanter Betriebsphasen entwickelt, die in ihrer Anwendung bisher vornehmlich auf den Einsatz in Personenkraftwagen unter Real-Driving-Emissions (RDE) Bedingungen beschränkt war. Das parallel entwickelte Projekt evTrailer hybridisiert Nutzfahrzeuge, indem der Trailer als elektrifizierte Komponente dient und damit eine hohe Variabilität der darstellbaren Betriebszustände des konventionellen Dieselaggregates der Zugmaschine erlaubt. Dieser Freiheitsgrad wird in dem vorgestellten Beitrag genutzt, um die mit Hilfe der MostRelevant-Methode identifizierten Betriebsphasen zu verschieben. Am Truck-in-the-Loop Prüfstand werden damit die entstehenden Emissionen unter Realbedingungen möglichst effizient reduziert.

Mit fortschreitender Entwicklung der Abgasapplikation auf Basis von realfahrtbezogenen Emissionsgrenzwerten wird zunehmend deutlich, dass ein emissionsminimaler Betrieb von Verbrennungsmotoren unter betriebswarmen Bedingungen technisch lösbar ist. Besonders relevant für die Gesamtemission ist damit das Erreichen und das Beibehalten jener betriebswarmen Bedingungen durch ein entsprechendes Thermomanagement. Dies gilt insbesondere für hybridisierte Antriebstränge. Deren gesteigerte Flexibilität in Punkto Betriebsweise führt allerdings zu deutlichen Aufwandssteigerungen bei der Applikation. Der zusätzlich vorgestellte Co-Simulations-Workflow beschreibt eine Herangehensweise, durch deren Einsatz den neuen Herausforderungen durch Modularität, Konsistenz und Echtzeitfähigkeit effizient begegnet werden kann. Kennzeichnendes Merkmal ist dabei die flexible Austauschbarkeit von virtuellen und realen Werkzeugen. 


\section{Einleitung und Motivation}

Im aktuellen politischen Kontext wächst der Wunsch nach Fahrzeugantrieben, die in allen

Betriebssituationen unter allen Randbedingungen die gesetzlichen Grenzwerte unterschreiten. Im Rahmen des Klimaabkommens aus Paris bedeutet dies eine Minderung der Tank-to-Wheel (TtW) $\mathrm{CO}_{2}$-Emissionen von Nutzfahrzeugen um $30 \%$ bis 2030 (1) bei gleichzeitiger Einhaltung der Sickoxidgrenzwerte $\left(\mathrm{NO}_{\mathrm{x}}\right)$. In diesem Kontext wird am Institut für Verbrennungskraftmaschinen und Fahrzeugantriebe der Technischen Universität Darmstadt (VKM TUD) eine Methodik zur Identifikation emissionskritischer Betriebszustände im realen Betrieb entwickelt. So können die größten Herausforderungen für die Abgasnachbehandlung auf der Straße erkannt werden und mittels neuer Antriebstechnologien Lösungen erarbeitet werden.

Zudem ist es anschließend möglich die erkannten Fahrsituation auf die Heavy-Duty Engine-in-theLoop (EiL) Motorenprüfstände des Instituts zu übertragen. Ziel ist es schließlich durch Anpassungen der Dosierstrategie des Reduktionsmittels oder durch Implementierung einer virtuellen Hybridisierung in der parallel zum Motor laufenden Echtzeitsimulation, das Emissionsverhalten des Motors positiv zu beeinflussen. In diesem Zusammenhang dienen erkannte, reale Emissionsevents als Grundlage für das Most-Relevant-Testing des neu entwickelten Antriebs.

In dem vorliegenden Beitrag wurde die herkömmliche Sattelzugmaschine mit einem elektrisch angetriebenen Auflieger (evTrailer) ergänzt. Die vorgestellte Betriebsstrategie des evTrailers kann zum Schmälern des Betriebstemperaturfensters des SCR-Katalysators eingesetzt werden. Nach oben wird die SCR-Temperatur durch Zugkraftunterstützung in Beschleunigungs- und Bergauffahrten und nach unten durch Lastpunktanhebung in Schubphasen begrenzt. Das Betreiben des SCRKatalysators in dessen optimalen Betriebsbereich bewirkt eine Minderung der $\mathrm{NO}_{x}-$ und $\mathrm{NH}_{3}-$ Emissionen. Ergänzend zu der gemessenen Zugkraft am Kingpin wird die SCR Temperatur als weitere Eingangsgröße für die Betriebsstrategie zurückgeführt.

Im ersten Teil des vorliegenden Beitrags wird auf die Erkennung kritischer Events aus realen Messungen eingegangen. Diese bilden die Grundlage für die im zweiten Teil vorgestellten Untersuchungen am Engine-in-the-Loop Motorenprüfstand der Technischen Universität Darmstadt. Die Potentiale zur Minderung der Schadstoffemission und zur Reduzierung des $\mathrm{CO}_{2}$-Ausstoßes mittels Hybridisierung von Lastkraftwägen mit zwei unterschiedlichen Betriebsstrategien werden im zweiten Abschnitt dargestellt.

\section{Identifikation kritischer Betriebszustände}

Zur Erfassung kritischer Betriebszustände im realen Fahrbetrieb wurde eine Sattelzugmaschine mit zusätzlicher Sensorik und Datenlogger ausgerüstet. Dies ermöglicht eine kontinuierliche Überwachung der motorischen und abgasnachbehandlungsrelevanten Größen während dem regulären Speditionseinsatz. Der verwendete Lkw hat eine tägliche Fahrleistung von ca. $800 \mathrm{~km}$ die er überwiegend auf Fern- und Überlandstraßen absolviert. Der Messzeitraum erstreckte sich über 18 Monate.

Die Identifikation emissionskritischer Fahrsituation aus der großen Menge an erfassten Daten erfordert eine gezielte Verarbeitung der Messungen. In diesem Zusammenhang wird am VKM TUD eine Methodik mit integrativen Ansatz entwickelt. Im Gegensatz zu einer schwellenwertbasierten Eventerkennung bietet dieser Ansatz die Möglichkeit, auch längere Events mit einem Emissionsniveau unterhalb des definierten Schwellenwertes zu erkennen. Vor der eigentlichen Eventerkennung werden die Fahrten in Abschnitte der Länge einer zuvor definierten Referenzarbeit unterteilt. Als Referenzarbeit wird die von dem im Versuchsfahrzeug verbauten Motors abgegebene mechanische Arbeit während eines WHTCs $\left(W_{\text {ref,WHTC }}\right)$ herangezogen.

Die realen erfassten Daten werden anschließend in $i$-Segmente die der Referenzarbeit $W_{\text {ref,WHTC }}$ entsprechen unterteilt und die prozentualen spezifischen Emissionen eines Abschnitts mit einem 
zuvor definiertem Grenzwert für Stickoxide verglichen. Dabei entsprechen spezifische Emissionen von $100 \%$ eines Segments genau dem zuvor definierten Grenzwert.

$$
\begin{aligned}
& W_{\text {Abschnitt }, i}=W_{\text {ref }, \text { WHTC }} \\
& \text { Grenzwert }_{\text {NOx }}=100 \%
\end{aligned}
$$

Abbildung 1 zeigt die Unterteilung einer 270 kWh Fahrt in neun 30 kWh Segmente sowie die prozentualen spezifischen Stickoxidemissionen jedes Segments bezogen auf den definierten Grenzwert.

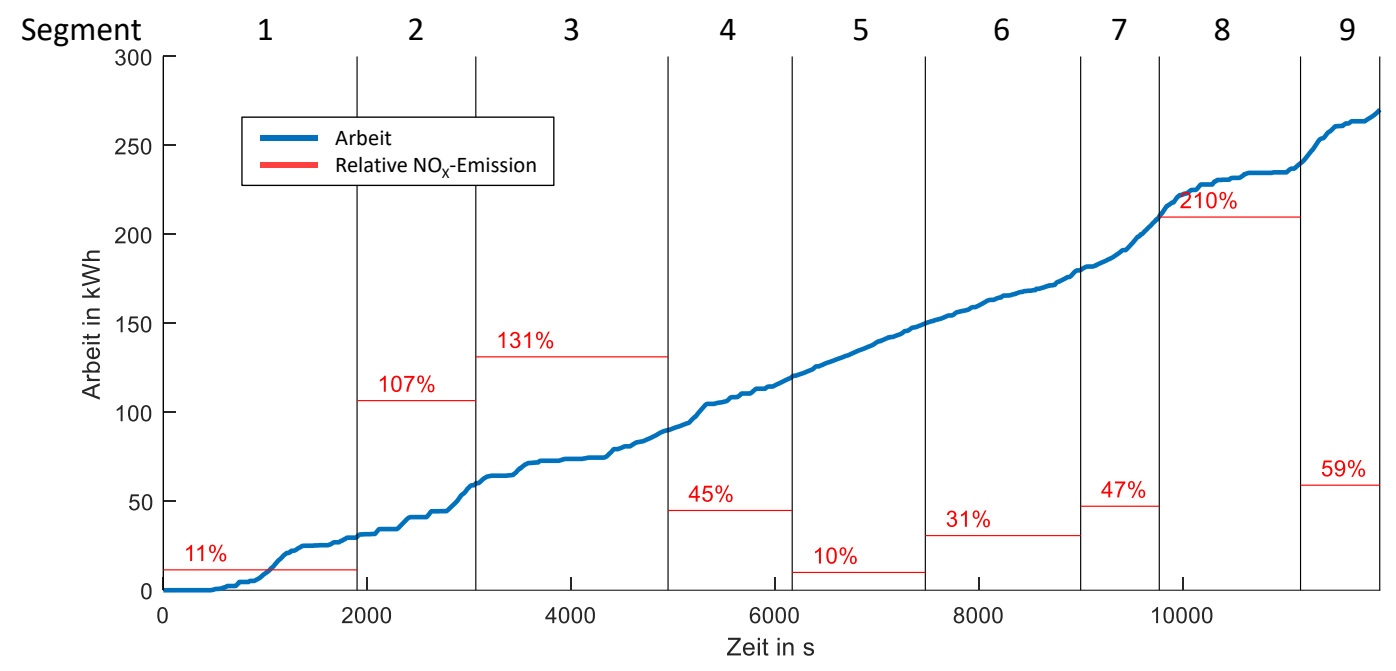

Abbildung 1: Unterteilung einer Fahrt in Segmente nach Referenzarbeit und zugehörige spezifische Emissionen

Übersteigen die spezifischen Emissionen eines Segments $100 \%$, so erfolgt die genaue Identifikation der Events innerhalb des erkannten Segments $i$ durch Analyse des Emissionsverlaufs. Mittels einer Nullpunkt-Erkennung werden die einzelnen Emissionspeaks des Segments isoliert. Abbildung 2 zeigt den zeitlichen $\mathrm{NO}_{x}$-Konzentrationsverlauf von Segment 8 aus Abbildung 1 mit der Erkennung einzelner Emissionspeaks. Nach der vorgestellten Peak-erkennung enthält das ca. 23 Minütige Segment 8 zwölf Emissionspeaks (a bis I).

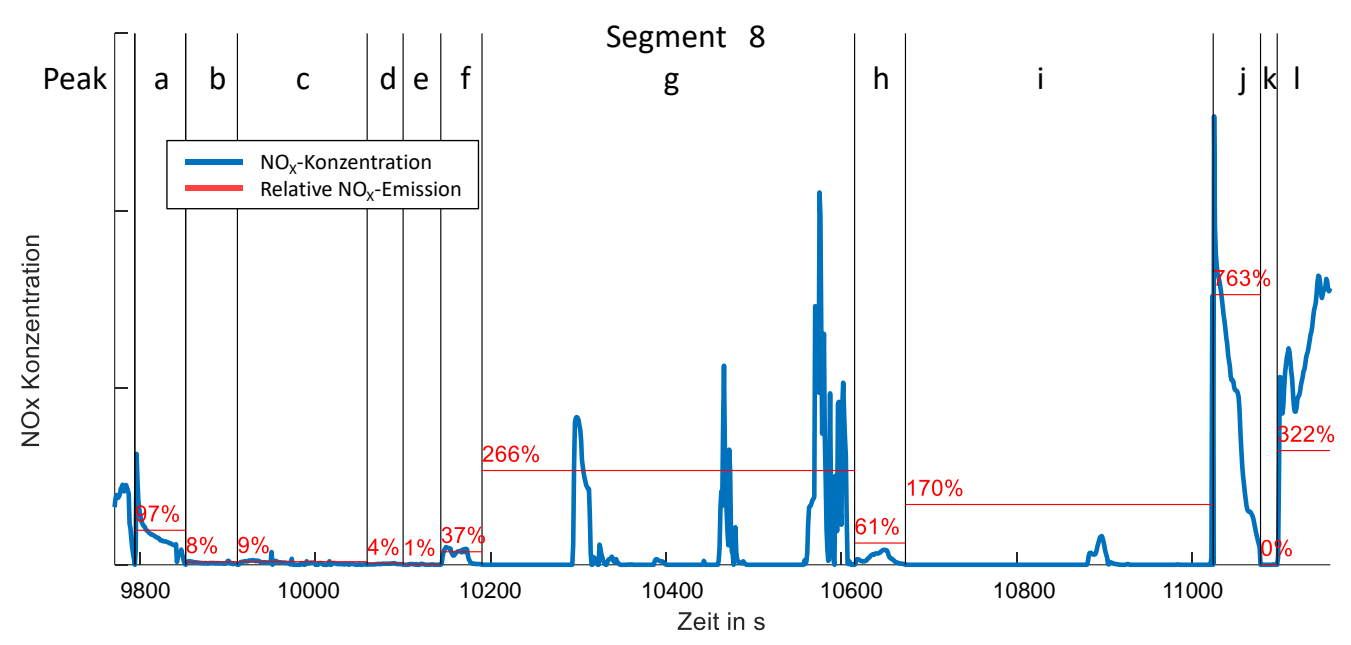

Abbildung 2: Emissionsverlauf mit Nullpunkterkennung zur Eventtyperkennung für Segment 8

Für jeden erkannten Emissionspeak werden erneut die spezifischen Emissionen berechnet und auf den definierten Grenzwert bezogen. Zudem werden für jeden Peak Kennwerte zur Einteilung nach Eventtyp berechnet. Hierfür sind zur Klassifizierung der Emissionsevents nach Fahrsituationen sowohl 
motorische und abgasnachbehandlungsbezogene Größen, als auch fahrdynamische und streckenbezogene Größen von Relevanz. Die Nullpunkterkennung ermöglicht eine zeitliche Zuordnung von Emissionen zu fahrzeug- und streckenbezogene Größen und hiermit eine Rückführung auf die Ursachen eines Events. Anhand der vorgestellten Methodik werden die identifizierten Emissionsevents Fahrsituationen und Zustände der Abgasnachbehandlung zugeordnet. Zudem ermöglicht der Bezug der spezifischen Emissionen auf den definierten Grenzwert während einem Emissionsevent eine Einteilung der Events nach Schwere und somit eine effiziente Analyse der Events.

Abbildung 3 zeigt die Einteilung der erkannten emissionskritischen Events mit der vorgestellten Methodik über den Messzeitraum von einem Jahr. Nach der motorischen Klassifizierung (Abbildung 3 Links) stellen die Situationen, in denen die SCR-Temperatur zu niedrig ist, zwei Drittel der kritischen Segmente dar. Eine hohe Leistung und somit hohe Raumgeschwindigkeiten bei hohen Temperaturen sind für ca. ein Viertel der erkannten Events verantwortlich. Die restlichen Events sind eine Kombination aus mehreren motorischen Ursachen und somit nach heutigem Stand nicht eindeutig klassifizierbar.
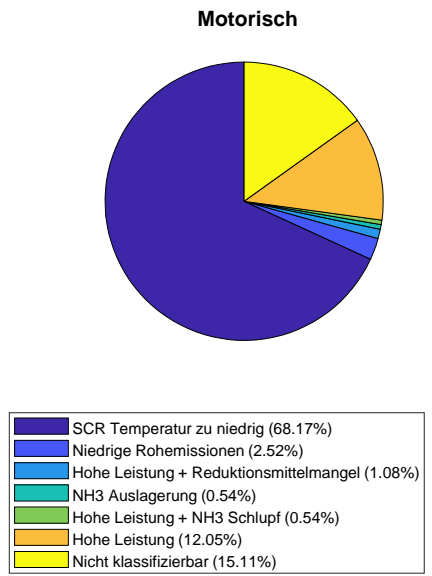
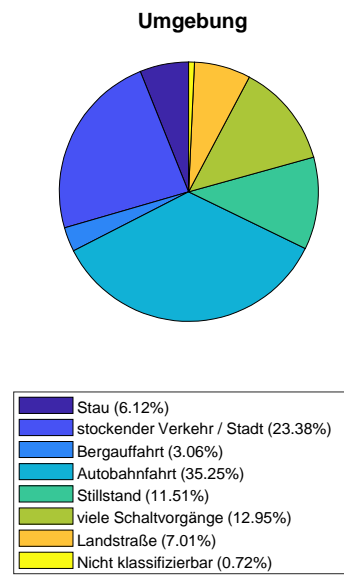
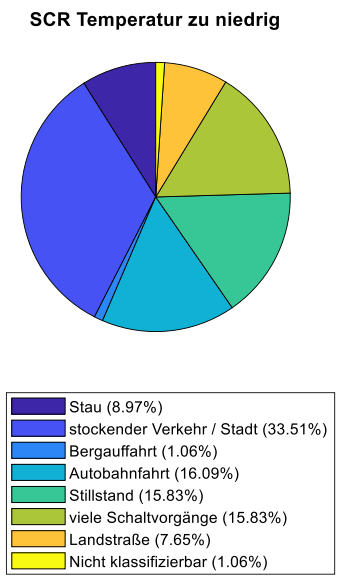

Abbildung 3: Klassifizierung der emissionskritischen Events nach motorischen und umgebungsbedingten Einflussparametern

In Abbildung 3 Mitte ist die streckenbezogene Klassifizierung der Events dargestellt. Die vorgestellte Eventerkennung detektiert einzelne Peaks, sodass anhand der zugehörigen geographischen und fahrdynamischen Größen die Fahrsituation eindeutig erkannt wird. Im vorgestellten Beispiel beträgt auf Grund des Einsatzprofils der Anteil an Emissionsevents auf Autobahnen $35 \%$, wobei $40 \%$ der Events auf Transientes Fahrverhalten zurückzuführen sind (Stau oder stockender Verkehr). Zuletzt stellt Abbildung 3 Rechts die streckenbezogene Einteilung bei zu niedriger SCR Temperatur dar. Die Hälfte der Events treten bei Stau, stockendem Verkehr oder im Stillstand auf. Die restlichen $50 \%$ kommen unter normalem Fahrbetrieb vor, zum Beispiel nach einer langen Bergabfahrt auf der Autobahn. Die Anzahl dieser Eventart könnte durch Erhöhung der SCR-Temperatur mittels weiteren Isolierungsmaßnahmen und Heizstrategien reduziert werden. Jedoch besteht im Gegenzug die Gefahr, bei hohen Lasten den Betriebsbereich des SCR-Katalysators nach oben hin zu verlassen. In diesem Kontext kann eine Hybridisierung den starken Temperaturgradienten, den zu hohen Temperaturen sowie der Abkühlung des SCR-Katalysators mittels Phlegmatisierung und Lastpunktverschiebung entgegenwirken. Zur Potentialbewertung des in diesem Beitrag präsentierten Hybridantriebs wird das Segment 8 aus Abbildung 1 auf den Engine-in-the-Loop Motorenprüfstand übertragen. Zur Konditionierung der Abgasanlage werden vor das Segment die vorherigen 30 Minuten der Fahrt hinzugefügt. Das Geschwindigkeits- und Höhenprofil aus Abbildung 4 dient im Folgenden als Strecke für die weiteren Untersuchungen dieser Arbeit. Auf der $79.7 \mathrm{~km}$ langen Strecke befinden 
sich drei Anstiege mit insgesamt 140 positiven Höhenmetern. Die mittlere Geschwindigkeit beträgt $78 \mathrm{~km} / \mathrm{h}$.

Am Engine-in-the-Loop Motorenprüfstand sind die Stecke, der Fahrer und das Fahrzeug in der parallel zum Motor laufenden Echtzeitsimulation implementiert. So ist eine Potentialabschätzung von noch

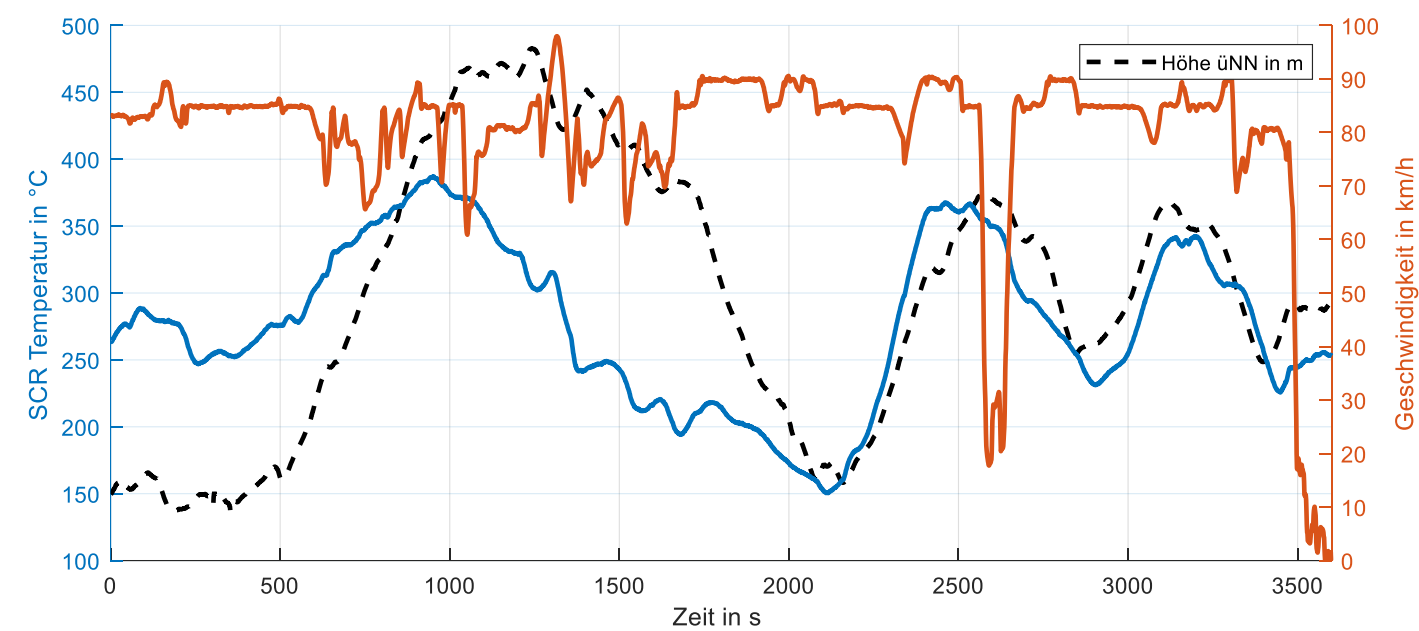

Abbildung 4: SCR-Temperatur, Höhenprofil sowie Geschwindigkeitsverlauf der ausgewählten Fahrt

nicht existierenden Antriebsstrangkonfigurationen bezüglich Stickoxidemissionen und Kohlenstoffdioxidausstoß möglich.

Im Folgenden werden drei Antriebskonzepte bei Kalt- und Warmstart auf der genannten Strecke untersucht. Dabei wird die konventionelle Sattelzugmaschine mittels evTrailer hybridisiert und zusätzlich noch um eine SCR-Temperaturregelung ergänzt.

\section{Hybridantriebskonzept: evTrailer}

Das in diesem Beitrag eingesetzte Nutzfahrzeug-Hybridisierungskonzept basiert auf dem im Rahmen des vom Bundesministerium für Wirtschaft und Energie (BMWi) geförderten Forschungsprojektes „evTrailer - autarkes elektrisches Antriebskooperationssystem für LKW-Trailer und Fahrzeuganhänger“ (2) entwickelten Antriebs. Dabei wird ein konventioneller Trailer mit zwei Elektromotoren sowie einer Traktionsbatterie und der zugehörigen Leistungselektronik versehen. Das elektrische System ermöglicht die Rekuperation von Bremsenergie bei Bremsvorgängen beispielsweise bei Bergabfahrten. Die gewonnene Energie kann anschließend zur Unterstützung des Antriebs in Phasen hoher Lastanforderung eingesetzt werden und reduziert so die vom Verbrennungsmotor benötigte Leistung und damit den Kraftstoffverbrauch.

Die Dimensionierung des im Boden des Trailers befindlichen Antriebsstrangs ist mittels mehrdimensionaler, multikriterieller Optimierung durchgeführt worden (3). Der in diesem Beitrag verwendete Antriebstrang besteht aus zwei Elektromotoren mit $75 \mathrm{~kW}$ maximaler Leistung und einer Batteriekapazität von $100 \mathrm{kWh}$, deren nutzbarer Ladehub aus Lebensdauergründen auf $50 \mathrm{kWh}$ reduziert wird. Der evTrailer ist als Plug-In System mit ladungsverbrauchender Betriebsstrategie vorgesehen, wodurch mittels externem Laden während Stillstandszeiten eine weitere Senkung der TtW $\mathrm{CO}_{2}$-Emissionen ermöglicht wird. 


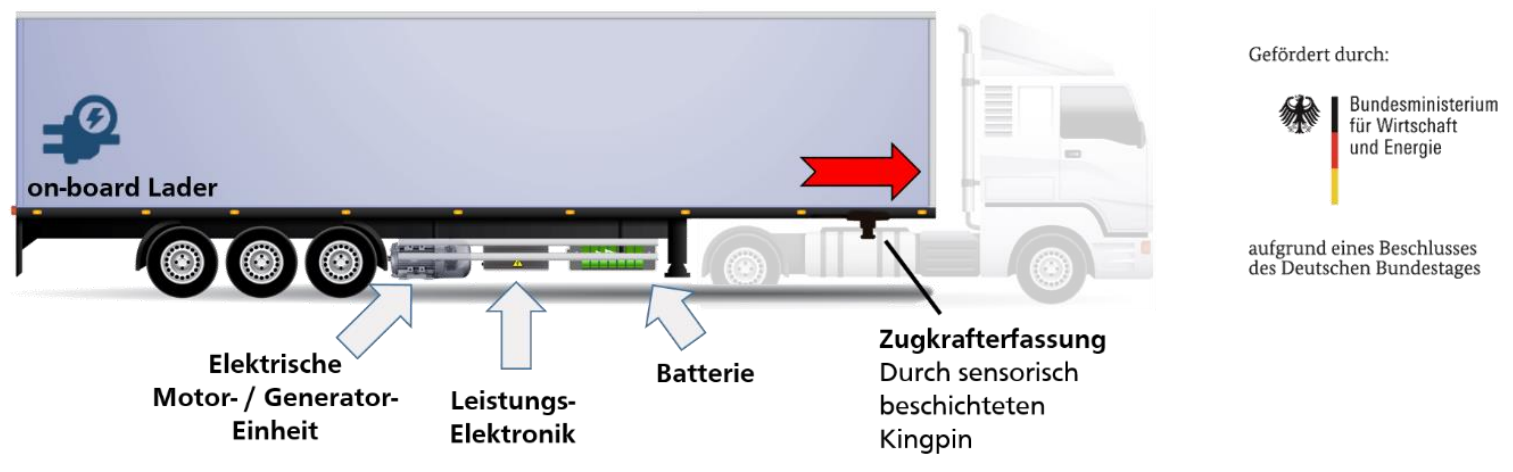

Abbildung 5: evTrailer Konzept

\section{Versuchsaufbau}

Vorhergegangene Untersuchungen (4) (5) haben gezeigt, dass die Hybridisierung von Verteilerfahrzeugen mit einer auf geringen Kraftstoffverbrauch optimierten Betriebsstrategie eine Reduktion des $\mathrm{CO}_{2}$-Ausstoßes um $30 \%$ bei gleichzeitiger Senkung der Stickoxid- und Ammoniakemissionen um 44 \% bzw. 30 \% ermöglicht. Ursache für die positiven Einflüsse auf die Schadstoffemission ist neben der Phlegmatisierung des Verbrennungsmotors die Reduktion der verbrennungsmotorischen Leistung durch die elektrische Unterstützung, welche wiederum zu niedrigeren Abgas- und damit SCR-Temperaturen führt und somit höhere Konvertierungsraten und niedrigeren Ammoniak-Schlupf zur Folge hat. Gleichzeitig wird so jedoch auch das Temperaturniveau in den Abschnitten reduziert, in denen sich das Abgasnachbehandlungssystem bereits beim konventionellen Fahrzeug im Bereich der unteren Dosiergrenze befindet. Die elektrische Unterstützung kann dadurch zu einem Unterschreiten der Dosiergrenze und somit zu erhöhter Stickoxid-Emission in diesen Situationen führen.

Daher wird in diesem Beitrag die vorhandene Betriebsstrategie um eine SCR-Temperaturregelung ergänzt. Die Temperatur des SCR-Katalysators stellt dabei die Regelgröße dar, während das Sollmoment der Elektromaschinen die Stellgröße des Systems bildet. Durch motorischen bzw. generatorischen Betrieb der E-Maschinen des evTrailers und dem daraus resultierenden Lastprofil des Verbrennungsmotors kann so der SCR-Katalysator in seinem optimalen Betriebstemperaturfenster gehalten werden.

Die Strecke aus Abbildung 4 wird als digitale Strecke inklusive Höhenprofil und Geschwindigkeitsbegrenzungen in IPG TruckMaker aufgebaut und bildet mit dem Fahrzeugmodell die konstanten Parameter auf der simulativen Seite. Zusätzlich wird das evTrailer-Modell parametriert und in IPG TruckMaker implementiert. Am Engine-in-the-Loop Motorenprüfstand wird lediglich der Verbrennungsmotor und das Abgasnachbehandlungssystem aus dem Fahrzeugmodell durch das am Prüfstand aufgebaute System ersetzt während die Simulation parallel auf dem Echtzeitrechner läuft. Der Fahrerwunsch aus der Simulation wird in Echtzeit dem realen Steuergerät am Prüfstand übergeben und die daraus resultierende mechanische Leistung dient der Simulationsumgebung zur Berechnung der erreichten Fahrzeuggeschwindigkeit. Mit dieser Vorgehensweise sind die Auswirkungen von Änderungen am Fahrzeug auf Emissionen und Verbrauch direkt am Prüfstand messbar. Die Untersuchungen befassen sich somit mit den drei folgenden Antriebsstrangkonfigurationen:

Konventionell: Sattelzugmaschine mit konventionellem Auflieger evTrailer: Sattelzugmaschine mit evTrailer evTrailer mit Temperaturregler: Sattelzugmaschine mit evTrailer und mittelsTemperaturregler erweiterte Betriebsstrategie 
Die drei Konfigurationen werden auf der vorgestellten Strecke unter Kaltstartbedingungen und mit einem auf $250^{\circ} \mathrm{C}$ vorgeheizten SCR-Katalysator verglichen.

In allen Versuchen wird nicht auf den vom Serien-Steuergerät gewählten Motorbetriebsmodus eingegriffen. Der Betriebsmodus des Motors ist somit lediglich von den Betriebsmitteltemperaturen und dem Zustand des Abgasnachbehandlungssystems abhängig.

\section{Versuchsergebnisse}

In Abbildung 6 sind die Temperaturverläufe der drei Antriebstrangkonfigurationen unter Kalt- und Warmstartbedingungen dargestellt. Die auf geringen $\mathrm{CO}_{2}-$ Ausstoß optimierte evTrailer

Betriebsstrategie greift unabhängig vom Zustand der Abgasnachbehandlung ein. Als Eingangsgrößen dienen dabei der Ladezustand der Batterie und das Vorzeichen der am Kingpin anliegenden Zugkraft. Die Betriebsstrategie des evTrailers mit Temperaturregelung hingegen berücksichtigt zusätzlich noch die SCR-Temperatur. Unterschreitet diese $235^{\circ} \mathrm{C}$, wird die Unterstützung des elektrischen Systems reduziert oder sogar im Generatorbetrieb dessen Batterie geladen, während der Verbrennungsmotor bei erhöhter Last betrieben wird. Hingegen wird, falls die SCR-Temperatur über $320^{\circ} \mathrm{C}$ steigt, die evTrailer-Unterstützung gesteigert insofern möglich, was zu einer Absenkung der
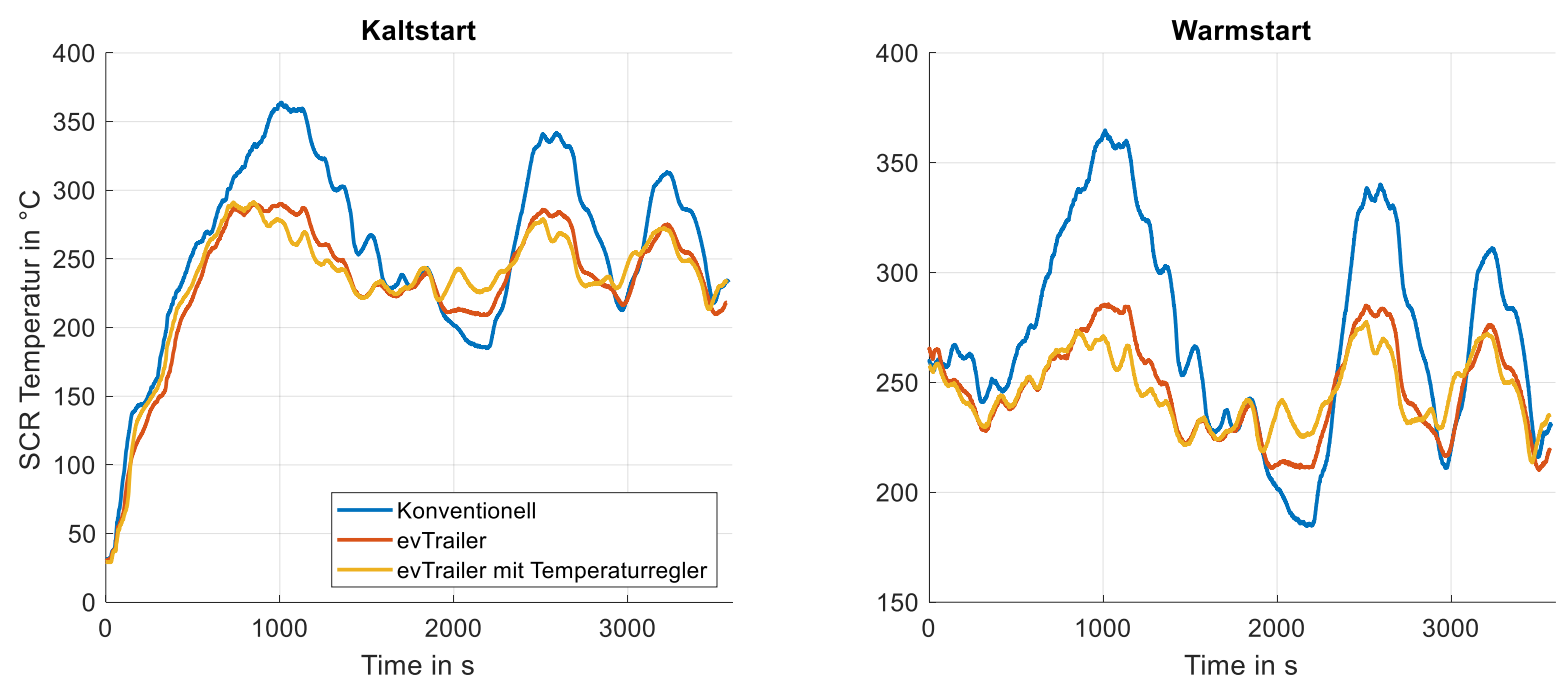

Abbildung 6: Verläufe der SCR-Temperatur

verbrennungsmotorischen Leistung und der Abgastemperatur führt. Die ausgewählten Grenztemperaturen für den Regler stammen aus Voruntersuchungen und liegen oberhalb der Abschaltschwelle der Reduktionsmitteldosierung bzw. unterhalb erhöhter Ammoniak-Desorption. Abbildung 6 zeigt, dass sowohl im Kalt- als auch im Warmstart die SCR-Temperaturspitzen über $300^{\circ} \mathrm{C}$ und die Temperatursenken unter $200^{\circ} \mathrm{C}$ bei beiden evTrailer-Betriebsstrategien vermieden werden.

Beim Vergleich zwischen den zwei evTrailer-Betriebsstrategien ist ab 1000 Sekunden sowohl im Kaltals auch im Warmstart ein Unterschied der Temperaturprofile zu erkennen: das Temperaturprofil des evTrailers weist eine größere Standardabweichung vom Mittelwert bei einem kontinuierlicheren Profil gegenüber dem Profil der temperaturgeregelten Betriebsstrategie auf. Das wellige Profil der erweiterten evTrailer-Betriebsstrategie ist die Folge des Reglereingriffs.

Die Auswirkung der zwei unterschiedlichen evTrailer Betriebsstrategien auf die Emissionen und den Kraftstoffverbrauch sind in Abbildung 7 zu sehen. Dabei wird der Betrieb mit konventioneller Sattelzugmaschine als $100 \%$ Referenz für alle betrachteten Größen angesetzt und die prozentuale Minderung gegenüber der Referenz dargestellt. 
Minderung in \% ggü. Konventionellem Antrieb

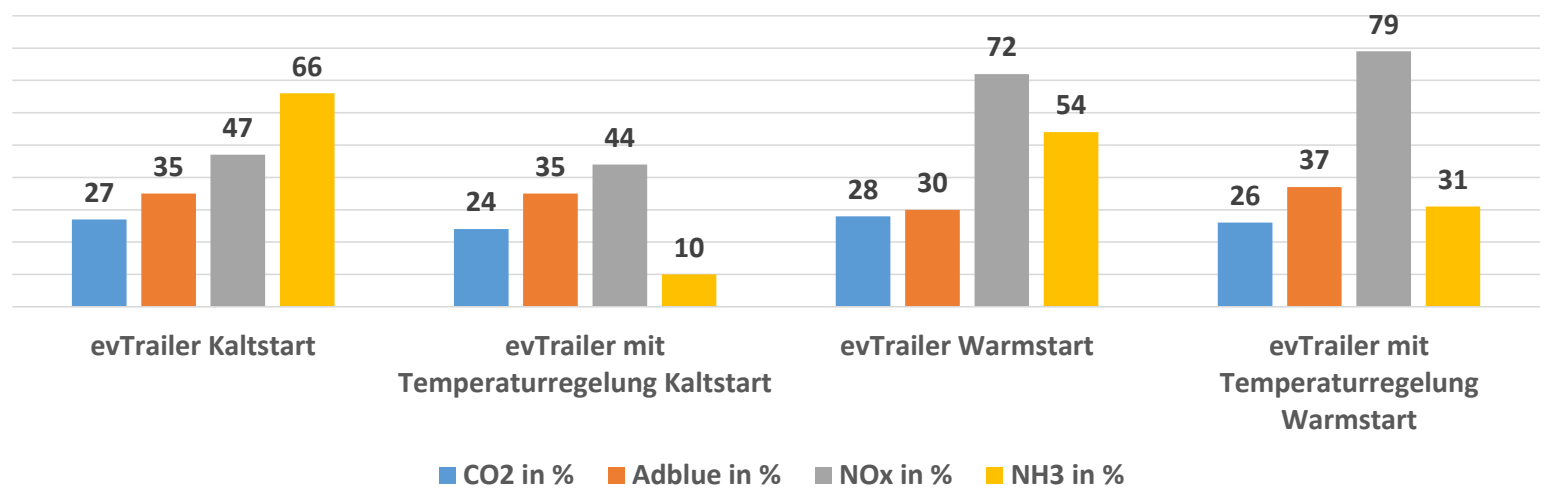

Abbildung 7: Minderung in \% gegenüber dem konventionellen Antrieb

Die blauen Balken aus Abbildung 7 zeigen das $\mathrm{CO}_{2}$-Einsparpotential einer Hybridisierung von Sattelzugmaschinen. Dabei weißt die auf $\mathrm{CO}_{2}$-optimierte Betriebsstrategie mit $27 \%$ im Kaltstart und $28 \%$ im Warmstart das größte Kraftstoffeinsparpotential auf. Im Kaltstart, ist die effektivere Reduktion der $\mathrm{NO}_{x}$-Emissionen gegenüber der Betriebsstrategie mit SCR-Temperaturregelung zum einen auf die um 30 s kürzere Aufheizphase und zum anderen auf die geringeren Temperaturgradienten zurückzuführen. Trotz den niedrigeren SCR-Temperaturen und dem gleichen

Reduktionsmittelverbrauch weist die temperaturgeregelte Betriebsstrategie im Kaltstart auch höhere $\mathrm{NH}_{3}$-Emissionen auf. Diese sind ebenfalls dem größeren Temperaturgradienten zuzuschreiben.

Das Einsparpotential der vorgestellten temperaturgeregelten Betriebsstrategie nach der Gesetzgebung (Kaltstart mit $14 \%$ und Warmstart nach $86 \%$ gewichtet) ist in Abbildung 8 dargestellt. Auffallend ist der Zielkonflikt zwischen $\mathrm{CO}_{2}$-Ausstoß und $\mathrm{NO}_{x}$-Emissionen: die maximale Kraftstoffeinsparung von knapp 28 \%wird mit dem evTrailer alleine erreicht während die effektivste $\mathrm{NO}_{x}$-Reduktion durch die temperaturgeregelte Betriebsstrategie erfolgt.

Durch Minderung der Rohemissionen erreicht die Betriebsstrategie mit Temperaturregelung eine

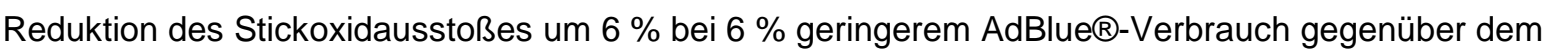
evTrailer.

Minderung in \% ggü. Konventionell mit Gewichtung (Kalt 14\%/Warm 86\%)

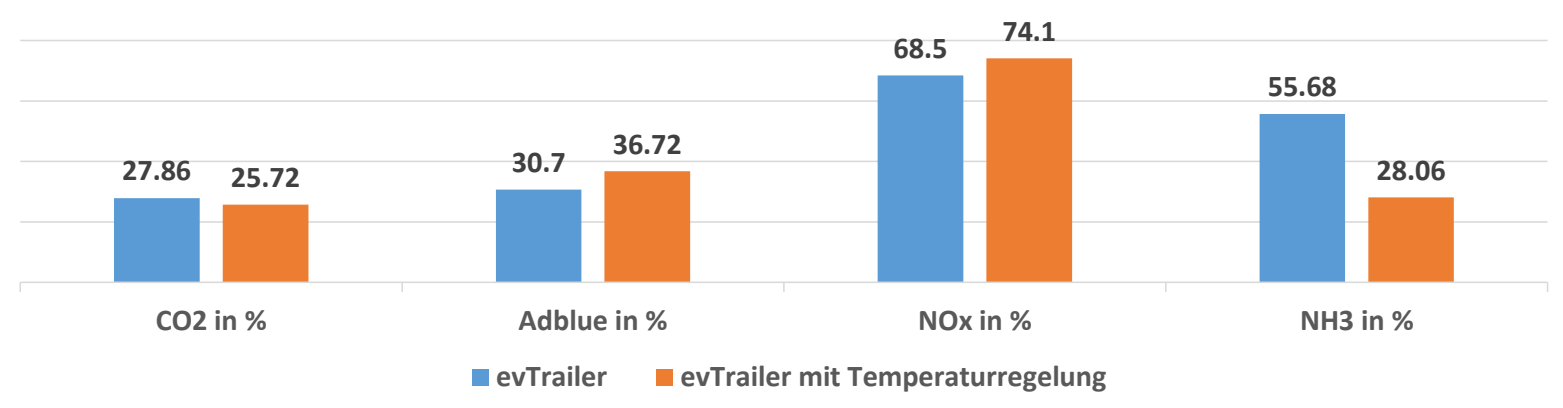

Abbildung 8: Gewichtete Minderung in \% der evTrailer-Betriebsstrategien gegenüber dem konventionellen Antrieb

\section{Zusammenfassung}

Der vorliegende Beitrag zeigt die Potentiale der Hybridisierung von Sattelzugmaschinen sowohl in Bezug auf Schadstoffemissionen als auch auf den Kraftstoffverbrauch. Zudem wurde gezeigt, dass die Verwendung der SCR-Temperatur als Eingangsgröße eines Reglers die Verweildauer des SCRKatalysators in ungünstigen Betriebsbedingungen reduzieren kann. Die Gegenüberstellung einer $\mathrm{CO}_{2-}$ optimierten Betriebsstrategie und einer Betriebsstrategie zur Minimierung der $\mathrm{NO}_{x}$-Emissionen weist 
auf den Trade-off der diesbezüglich getroffen werden muss. Zuletzt wurde gezeigt, dass die elektrische Unterstützung des Verbrennungsmotors sowohl auf Emissions- als auch auf der Kraftstoffseite einen Vorteil hervorruft.

Bei der Auswertung der Ergebnisse fällt jedoch der negative Einfluss von starken Temperaturgradienten im SCR-Katalysator auf. Die hohen Gradienten haben besonders im Ammoniakschlupf einen negativen Einfluss. Die Ergebnisse zeigen zudem, dass eine weitere Reduktion der Stickoxid-Emissionen während der Aufheizphase des Katalysators möglich ist.

\section{Literatur}

1. EUROPEAN COMMISSION. Proposal for a REGULATION OF THE EUROPEAN PARLIAMENT AND OF THE COUNCIL setting CO2 emission performance standards for new heavy -duty vehicles, 2018.

2. evTrailer Autarkes elektrisches Antriebskooperationssystem für LKW-Trailer und Fahrzeuganhänger [online], 2019 [Zugriff am: 1. Februar 2019]. Verfügbar unter: http://evtrailer.de/

3. DAUTFEST, A., M. THIEM und BEIDL, UNIV.-PROF. DR. TECHN. CHRISTIAN BEIDL, Hg. Entwicklung eines Batteriesystems für elektrifizierte LKW-Anhänger, 2017.

4. MIKULA THIEM und NICOLAS HUMMEL, PROF. DR. TECHN. CHRISTIAN BEIDL. Betriebsbedingungen für Systeme zur Abgasnachbehandlung im hybridizierten Nutzfahrzeug. In: MTZ, S. 94-101.

5. MIKULA THIEM. Impacts of powertrain hybridization on engine exhaust behaviour of heavy duty vehicles with electrified trailers. In: 6. Internationaler Motorenkongress Baden-Baden. 